\title{
Wireless Body Area Networks: Challenges, Trends and Emerging Technologies
}

\author{
Bogdan Antonescu \\ Department of Electrical and Computer \\ Engineering \\ Northeastern University \\ Boston, MA 02115, U.S.A \\ antonescu.b@husky.neu.edu
}

\author{
Stefano Basagni \\ Department of Electrical and Computer \\ Engineering \\ Northeastern University \\ Boston, MA 02115, U.S.A \\ basagni@ece.neu.edu
}

\begin{abstract}
We provide a comprehensive review of the challenges and emerging technologies for WBANs: Wireless Body Area Networks. We describe leading applications and the tiers of the WBAN architectures, itemizing their major design areas. A succinct review of solutions, progress and possible future research in the areas of sensors, power and radio hardware components, the network stack, localization and mobility techniques, security and privacy, and of certification and standardization follows. We finally make the connection between WBANs and the fast growing research field of Cognitive Radios, which help establish efficient wireless transmissions in an increasingly crowded radio spectrum.
\end{abstract}

\section{INTRODUCTION}

Wireless Body Area Networks (WBANs) is a field developed in the past 15 years as a result of applying Wireless Personal Area Networks (WPAN) to the communications on, near and around the human body. This was possible due to research advancements in wireless sensors design and miniaturization, low-power sensor circuitry, signal processing and communications protocols. The field shares many of its challenges with the more general one of Wireless Sensor Networks (WSNs) [20]. Nevertheless, there are many differences between the two, especially with respect to deployment, density, data rate, latency and mobility [4]. Sensors in WBANs are less dense because there is no need of redundant nodes. The nodes also exhibit a fairly constant, more periodic and predictable data rate, and their latency can be traded off for improved reliability and decreased power consumption, based on specific applications. They can also be considered very mobile in comparison with the usually high staticity of WSN nodes. The applications of WBANs are numerous, ranging from health-care and telemedicine up to fitness and sports training, interactive gaming, and personal information sharing and authentication. WBANs can also be deployed in life-threatening environments to help protect soldiers, first responders and deep-sea or space explorers.

Permission to make digital or hard copies of all or part of this work for personal or classroom use is granted without fee provided that copies are not made or distributed for profit or commercial advantage and that copies bear this notice and the full citation on the first page. To copy otherwise, to republish, to post on servers or to redistribute to lists, requires prior specific permission and/or a fee.

BODYNETS 2013, September 30-October 02, Boston, United States Copyright (c) 2013 ICST 978-1-936968-89-3

DOI 10.4108/icst.bodynets.2013.253722
This paper is meant to provide a succinct yet comprehensive account of the challenges of WBAN research, and of the most recent technologies and techniques proposed to meet those challenges. In particular, and differently from previous survey accounts, we want to give an overview of trends in networking research that can progress WBANs into safe and useful technology.

The paper is organized as follows. In Section 2 we sketch a high-level view of WBANs applications. Section 3 presents the WBAN general architecture, and itemizes their design and implementation challenges. Section 4 elaborates along the implementation issues for both WBAN application and architectures. Section 5 bridges WBANs with the new emerging field of Cognitive Radios and underlines the importance of establishing radio communications using this technology. Conclusions are drawn in Section 6.

\section{APPLICATIONS FOR WBANS}

The most obvious application of WBANs is in the medical field, for healthcare and patient monitoring. The new concepts of m-Health [33] and 4G-Health (through 4G mobile communications) [32], combined with the Internet of m-Health Things (m-IoT) [31], enable new venues and outcomes for telemedicine and a personalization of the future m-Health services (i.e., p-Health [57]) through medication protocols and drug delivery tailored to the specific genetic baggage of each person. Long-term research for the use of WBANs in military and public safety [28] has seen two benefits. In battlefield situation, it is important to monitor vital signs of soldiers and stress levels induced by heat or other factors, to asses the physical and mental performance of the troops [34]. The other advantage of monitoring vital signs is a much faster triage of wounded soldiers. The complex, unstable and very dangerous environment of the battle field raises not only challenges related to the design of sensors (e.g., textile-based wearable electronics [63], bodycentric antennas [16]), but also communication security issues fostering novel ideas for cryptographic key generation and distribution, such as fuzzy vault cryptographic primitives used in conjunction with various parameters measured by WBAN sensors [59]. All these also apply to the case of first responder teams deployed at disaster sites. In sports and fitness monitoring applications, the real-time information received on athletes' heart rate, temperature, body position or impact during a game may identify critical situations. Thus, WBANs prevent burn-outs and injuries and maximize the performance of the athlete. Motion analysis performed by connecting the sensory information with com- 
puter vision techniques can also provide an optimum movement advice to patients who suffered a sports injury and are now in recovery [8]. This service is equally useful for monitoring the condition of elderly patients: Imagine a home rehab scenario in which the patient can perform exercises under the supervision of a computer [58]. As a last application of WBANs, computer gaming and entertainment evolved so much through the past decade because of the interactive virtual reality entertainment. To help create this virtual reality, accelerometers, gyroscopes and magnetic field sensors were combined in wearable inertial measurement units, to reconstruct the exact motion trajectories of body parts [41].

\section{WBAN ARCHITECTURE}

The general architecture of the WBAN network has three tiers [12]: Intra-WBAN, inter-WBAN and beyond-WBAN (Figure 1).

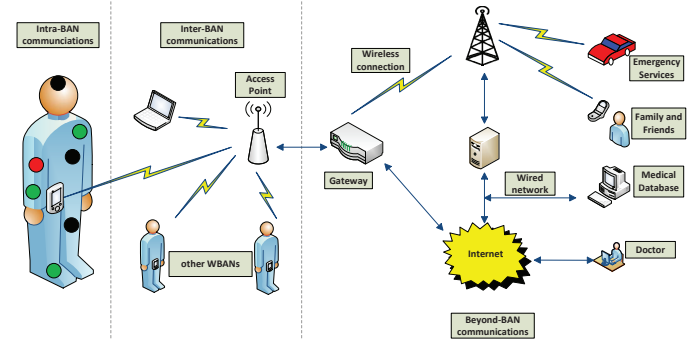

Figure 1: A three-tier architecture for WBANs.

The intra-WBAN refers to two types of communications: Those between body sensors and those between sensors and a portable Personal Server or Device (PS/PD), also called Body Control Unit (BCU), Body Gateway or Central Node. The sensors can be on, near or within the body, and they are of two types. Some of them only respond and collect data on physical stimuli, and then process and report this information wirelessly to the portable PD. Some others called actuators perform medicine administration based on the information received from other sensors in the same network, or through interaction with the user. The PD is the one that gathers all the information acquired by sensors and actuators. Once this information is collected by the PD, it has to be communicated through one or more access points (AP) to other networks that are easily reachable (e.g., Internet or cellular networks): This is the inter-WBAN communication. The communication standards deployed include Bluetooth/Bluetooth Low Energy, ZigBee, UWB, cellular and WLAN. A gateway device makes the connection with the beyond-WBAN communication network, thus increasing the coverage range and allowing authorized health-care personnel to access remotely the patient's medical information through Internet or cellular networks.

The major design areas relevant for the WBAN architecture are: 1. Sensors, power and radio hardware components, concerning sensors design, location and positioning of nodes, signal processing, data storage and feedback control, power source, dynamic control and energy conservation, energy harvesting technologies, and antenna design. 2. Network stack for radio and wireless transmissions, channel modeling, interfaces with other wireless transmission standards, interference, efficient MAC protocols, and cross-layer approaches. 3. Localization and mobility, concerning mobility awareness protocols, location and positioning of nodes. 4. Security and privacy is about secure data collection, processing and storage, security and integrity of data transmission, and invasion of privacy. 5. Certification and standardization takes care of dependability and QoS issues, service-oriented architecture (SOA), interoperability, certification and regulatory processes.

\section{WBAN IMPLEMENTATIONS: DESIDER- ATA AND CHALLENGES}

We identify and describe the main challenges to the implementation of the components of the WBAN architecture, citing solutions, progress and possible research in the corresponding research fields.

\subsection{Sensors, Power and Radio Hardware Com- ponents}

Body sensors: Tasks and requirements. The main design requirements for any "body sensor" node are to be extremely small and thin, non-invasive, capable of wireless communication and to take as less power as possible. Ideally, its battery would last for many years or, even better, energy harvesting techniques would enable the nodes to operate "perennially" [5]. The body sensor has to perform few tasks, including collection of the physiological signal, processing and storage of acquired information and data transmission (to PD) using its radio. Sensors can be grouped into three main categories: Physiological sensors that measure various vital body signals (e.g., blood pressure, temperature, blood oxygen, ECG, EEG, EMG); biokinetic sensors that measure acceleration and angular rate of rotation based on human body movement, and ambient sensors that measure environmental parameters (e.g., temperature, pressure, light, humidity, sound levels). Since WBANs do not usually have redundant nodes, ineffective placement or unintended displacement of nodes can render the entire process of data collection useless. Therefore, signal classification/qualification is very important right there on the node platform before data streams are sent to the PD.

Signal processing. Information extraction from monitored transient events or from trends (e.g., fever onset) proves that a certain level of signal processing on-the-node is advantageous because it reduces the in-network data rate. A trade-off between processing and communication is required to meet real-time requirements. To optimize this process, some methods consider context data awareness and predictive models combined with dynamic voltage-frequency scaling. If signal processing implies feature extraction algorithms and classification methods, on-node storage is also relevant for saving battery life. Caching data is also good for signal classification because the node might "learn" (by storing patterns) to classify healthy signs from pathological ones. Therefore, the challenge here is to explore low-power non-volatile memory devices like MRAM and RRAM, and to embedded them in the tiny sensor platforms.

Data transmissions. Nodes need to communicate with each other or with the PD. Unlike WSNs, in WBANs the communication channels are heavily attenuated and shadowed by the human body. To preserve quality of service (QoS), techniques including adaptive channel coding, dynamic power adjustment, MIMO, and QoS-aware MAC should be used. UWB is also widely considered in [66] and [23]. The use of newer technologies involves: Smart textiles [42] that embed sensing and wireless capabilities in clothing, energy-aware 
routing protocols [36], near-field communications [2] that suffer path loss less than the more traditional radiated communications, and body-coupled communications (BCC) [3] that use the human body as transmission channel at frequencies below $100 \mathrm{MHz}$, thus eliminating complex modulation schemes and high-frequency front-ends.

Power source and conservation. Lithium-ion batteries cannot pack enough capacity in a small volume. One other choice for energy storage is through super-capacitors and carbon-nanotube-based devices [24] that are, however, not yet widely available. One example is a mesh fabric (Power Felt) comprised of tiny nanotubes that convert body heat into energy [25]. Other emerging solutions for re-charging sensor batteries are given by energy harvesting devices that collect energy from various sources, external to the node [5]. The two main competing technologies suitable for WBAN nodes are inertial energy harvesting devices and thermoelectric devices. According to a theoretical model [43] for a certain volume, the kinetic device is superior, but the thermal device has a $70 \%$ effectiveness in achieving the forecast power density values comparing with only $1 \%$ efficiency for the inertial device. Yet another way to transfer energy to recharge the sensor batteries is through resonant evanescent waves $[38,15]$, the challenge being to apply it to the shortrange transmissions of WBANs.

Antenna design. Antenna design is possibly one of the most difficult tasks in a WBAN architecture because it is very important for the reliability of communication. It also has a significant effect on some of the intrinsic parameters of the network like power consumption, capacity, range, BER, and security. The antenna is affected by the weight, posture and even type of skin of the person wearing the WBAN node. Its shape and size depend on the location of the node and on the radio technology used for transmission; for this reason, heating effects and maximum power are two major concerns. Some of the primary challenges for its design are: Gain, bandwidth, pattern characteristics (e.g., polarization), and decreased sensitivity because of the environment. Equally important is the ability to connect efficiently two nodes that are not in line-of-sight, i.e., to propagate trapped surface waves called creeping waves. In connection with UWB transmissions, a thorough analysis of multipleantenna UWB systems and beamforming would be in order, because they increase the SNR levels at the receiver.

\subsection{Network Stack}

Radio technologies and channel modeling. WSNs, and especially WBANs, need a reliable link with the lowest power consumption possible. Among the design aspects and parameters that need optimization we enumerate the node transmission power, the antenna gains, the interaction between antenna and the human body, the path loss, the SNR levels, and the fade margins. Interference from other wireless devices sharing the same spectrum is another important parameter to consider, since it often results in degraded performance and lower energy efficiency [17]. Deep fading also occurs when a received signal is highly obstructed by physical obstacles. For a sensor implanted in the human body, the low power radio signals do not propagate easily, and they are attenuated and distorted even more based on the different tissues they encounter. To solve these problems, cooperative mechanisms from WSNs are adapted to WBANs [45]. These mechanisms combine various tech- niques like relay channels, multi-hop communications, and virtual MIMO or multi-sensor MIMO. Coding schemes and synchronization are required to attempt to obviate the outside interference from nearby WBANs. Electromagnetic propagation modeling of the wireless radio waves on and inside the human body is used in [22] to study the transmission channel with the goal to determine which propagation model takes place (i.e., diffraction, reflection, transmission, surface waves). Diffraction seems to be the main propagation mechanism around the human body [67].

Non-RF transmissions. Wireless communications based on $\mathrm{RF}$ electromagnetic waves do not have a good propagation through the human body because signals get attenuated by body tissues. In-body transmissions are required to be lowpower, so heat and cell death do not become an issue. As a totally novel research, in $[18,52]$ a new medium for communication inside the body is proposed: Ultrasonic waves. Identified challenges are related to transmission frequency and power, bandwidth and transducer size. Reflections and scattering are the main concerns, together with multipath fading resulting from delayed versions of the transmitted signal arriving at the receiver because of the human body non-homogeneity. Another non-RF transmission is called body-coupled communication (BCC): Galvanic and differential capacitive coupling are analyzed in [62] and [53], respectively. Since communication is limited to the proximity of the body, body shadowing is not as important as in the RF-based WBANs. Nevertheless, interference is a problem when electronic equipment is present in close proximity, so one might think that spread spectrum modulation schemes (Direct-Sequence or Frequency-Hoping) could work better.

Energy-efficient MAC protocols. A Medium Access Control (MAC) is at the core of any communication protocol stack and affects Quality of Service (QoS) provisioning. Its job is to attenuate the effect of collisions and to achieve the maximum possible throughput with a minimum latency, thus increasing network communication reliability and performance, and maximizing energy efficiency. Many energyefficient MAC protocols have been proposed and researched for WBANs, ranging from the work of [12] that shows basic techniques for generic WSNs (e.g., radio is turned on/off periodically, low-power listening protocols with channel polling, scheduled channel polling to synchronize all nodes) to achieve low throughput and delay. Challenges to energy efficiency in WBAN MAC protocols are identified in [19]. These, include collisions, overhearing, and idle listening. Examples of contention-based and contention-free MAC protocols introduced in literature are provided in [19]. The main advantage of a CSMA/CA-based MAC is its simplicity, low delay at low/medium traffic and reliability of transmissions at the expense of higher power consumption. On the other hand, a TDMA-based MAC is more efficient power-wise, but is not very flexible and scalable, and has the major requirements of having the node synchronized. Another technique to reduce the idle listening, a major culprit of energy consumption, involves an out-of-band communication channel, i.e., a secondary radio channel used to wake up the node scheduled for transmission [21]. In [30], TDMA is combined with a multi-beam antenna present at the node coordinator (PD), and provides what is known as Spatial Division Multiple Access (SDMA). The advantages of using multi-beam directional antennas include spatial reuse, extended range 
and energy saving, plus reduced delay because during one time slot the central node can receive transmissions from all sensor nodes. If idle listening and overhearing are not an issue, the challenges of this method shift towards the design of directional antennas [37].

Routing protocols. In order to reach the body central node (BCU), some packets might have to transit through some intermediate node, that would act as a relay. So, the reasonable question is which multi-hop routing scheme should be used. As in general WSNs, energy aware routing is a viable answer, that copes well with the problem of battery depletion of the WBAN nodes. Furthermore, routing solution should consider the challenge of mobility, in that it might induce channel fading and increased BER, partly because of the body movements and partly because of the interference with other nodes from different WBANs. Two types of routing protocols have been identified in [64], namely, cluster-based and flat-based. Another way to produce the optimal routing path is through minimum spanning trees as explored in $[6,39]$ where the tree is periodically and dynamically re-arranged based on the energy ratio between the initial battery power and the remaining power of each node. Excessive data processing and communications produce heat that can damage surrounding tissues. Thermal aware routing protocols have been introduced for this purpose [55]. The main desiderata is finding a model that captures the temperature increase and takes into account the identification of heat sources, to route the packets suitably.

Cross-layer approaches. There is a desire to analyze and understand the inter-dependencies between protocols at different layers of the stack in order to optimize the overall system performance. Since transmissions are a major consumer of energy in WBANs, smart signal processing at the node level could help decrease consumption, so upper layers should take care of the aggregation and compression of data, depending on the application type (i.e., streaming or monitoring). The Transport Layer needs advanced error detection schemes, while the Network Layer has to carefully consider various topologies (centralized vs. mesh) and to come up with a traffic profiling mechanism, while making channel access choices jointly with routing ones. Other issues include optimizing burst traffic and the overall end-to-end latency, especially when the WBAN is integrated with other wireless transmissions standards (e.g., GSM) for data transmission outside the WBAN. Finally, at the Data-Link Layer, energy can be saved through a multitude of power-aware $M A C$ protocols based on different strategies (e.g., fixed or variable sleep cycles, opportunistic packet transmissions), all of them cognizant of the latency they induce. Thus, the goal of a cross-layer approach is to exploit the dependencies between different layers and to produce a protocol able to handle multiple tasks. One such example is provided by WASP (Wireless Autonomous Spanning Tree Protocol) [6] and by its improved version CICADA (Cascading Information retrieval by Controlling Access with Distributed slot Assignments) [40]. The protocol handles both the medium access control and the routing by using a spanning tree to route data from nodes to sink, and by allocating time slots to different nodes in a distributed manner.

\subsection{Localization and Mobility}

Location/context awareness. WBANs can interact with other WBANs or nearby WSNs. Since sensed data can be related, it makes sense to group them, possibly merging them, depending on the specific situation that generated them. This is called context awareness. Another use of WBANs concerns being able to localize persons who might need immediate help. Localization is enabled by cooperative localization algorithms that extend the usual measurements for localization to nodes with unknown location. A classification of cooperative localization algorithms is given in [47] where they are partitioned into centralized and distributed algorithms. We are interested in distributed algorithms because we might not have a central processor with enough computational power to perform all calculations required for producing coordinates. The use of Bayesian networks has also been advocated for efficient sensor location [13]. In this context, mobility is the challenge. Locating and tracking sensors in real-time, in a network with energy constraints, is a distributed detection problem. To solve it, we can adapt the Sequential Monte Carlo (SMC) method for the localization of wireless sensors, as proposed in [29].

\subsection{Security and Privacy}

Data security and privacy. The security, integrity and privacy of the data gathered from a monitored person are key aspects of data transmission and processing in a WBAN. In [50], the topic is presented in connection with the limited energy supply and computational resources available in these networks. With respect to data storage, a secure WBAN should ensure confidentiality, integrity, availability and dependability; data must be encrypted and kept confidential during storage. Data cannot be modified illegally, should always be available to health-care personnel, even under denial-of-service (DoS) attacks, and should be retrievable even if the nodes fail. With respect to data access, the secure WBAN should provide services like privacy, non-repudiation, revocation, and accountability; only certain people can access the data, and the origin of data is known and non-disputable. Finally, with respect to data transmission, the traffic in a WBAN should always be encrypted, authenticated and impossible to be modified/forged in transit to destination without being detected. Therefore, we need authentication, confidentiality, data integrity and freshness. Also, a secure key distribution is at the core of the key management, and is required in order to provide valid and certified key material to the implemented crypto mechanisms. Communications between sensors in different WBANs can easily cross over when people get in close proximity of one another, so sensors need to know to what network they belong. This is a matter of node authentication that papers like [49] described from a novel point of view: Biometrics. This technique can solve not only the user authentication problem, but also the secure key distribution by implementing a fuzzy commitment scheme [35].

\subsection{Certification and Standardization}

Quality of Service $(Q o S)$. The QoS of traditional data networks cannot be applied directly to WBANs. Researchers adopted and modified the QoS requirements for WSNs. These requirements were grouped with respect to each layer in the OSI protocol stack [61]. Challenges and limiting factors that need to be considered for satisfying the QoS in WBANs are summarized by [1], and include resource limitation, unpredictable traffic patterns, network instability, network dynamics, energy balance, data redundancy, heterogeneous traffic types, packet criticality, unbalanced traffic, 
and multiple collection points. Besides these factors, there are some others like asymmetric network architectures with many different radio technologies that pose the question for a "star-shaped," adaptive QoS.

Interference. Interference is another powerful factor that can affect the performance of WBANs significantly. Nodes are close to each other and also influenced by the human body. In addition, they are also subject to outside sources of noise and perturbations from electronic equipment that surrounds us in daily life. Therefore, we classify interference as within the WBAN (the influence of human body on heterogeneous nodes that try to communicate at the same time with the BCU), inter-user interference [14] (WBANs communicate independently, but in close proximity of each other, as in patients wearing WBANs in hospital settings), and interference from communication equipment in the vicinity of WBANs [44] (e.g., WiFi and Bluetooth office/home equipment). The challenges for these types of studies are split in two categories. First, we need a set of measurements for predicting and modeling the chance of interference (e.g., distance from a source of signal, RSSI, path loss, multipath, amplitude distortion, jitter). Second, we need to find a set of parameters that help quantify the level of interference.

Dependability. Dependability is yet another challenge for WBANs that has not received much attention. It is a combination of reliability, security and availability that envisions all three branches of WBAN communication: Inter, intra and beyond-WBAN. According to [26], dependability is a trait of a system that describes its consistent behavior when it provides a service with minimum faults. Six properties that a WBAN should exhibit in order to be deemed dependable are mentioned in [54]: Reliability, availability, safety, confidentiality, integrity, and maintainability. In [27], all potential failures for an undependable WBAN are identified, along with their causes, effects and possible recoveries.

Service Oriented Architecture (SOA). So far, we have described the various challenges related to building and interconnecting tiny body sensors. General aim is that of maximizing their overall efficiency and decreasing their power consumption, in order to make them more practical and "wearable". One other challenge to make them even more accepted for large use would be to transform this network of devices into a network of services following what is called a Service Oriented Architecture (SOA). For the WBAN architecture presented in this paper, all SOA features translate into a middle layer that separates the sensor and communication functions from the application layer. Some of the reasons for this separation are easier integration and control of various heterogeneous sensors, simple network configuration mechanisms, and the chance to incorporate other services in the future [7]. The standardization of messages exchanged among these services will also increase interoperability and provide a higher scalability.

\section{TOWARDS COGNITIVE WBANS}

Understanding and interpreting complex human movements, rather than only fragments or gestures will allow automatic monitoring of user activities. This concept of context awareness [41] can interconnect many activity-aware systems, to provide crowd behavior and thus useful information to first responders in emergencies or disaster cases. The new cloudbased computing architecture opened the path for a new set of applications where small devices like WBAN nodes can communicate with data-intensive programs like augmented reality [56]. In medical applications, WBANs have a great potential to help people in rural areas where there is a demand for high-quality healthcare services with limited financial resources. Thus, WBANs shift the centralized inhospital healthcare to a more advanced in-home healthcare. To achieve these goals, a new concept of Utility Grid Computing (UGC) is meant to supply what the small WBANs lack: Access to the appropriate computational services and the high-bandwidth to a large array of distributed resources [46]. One important issue in WBANs is the interference among transmissions in the very crowded radio spectrum. In order to improve the efficiency of wireless communications in WBANs, an emerging technology like Cognitive Radio (CR) has been proposed [48, 10]. The CR idea to observe, learn, decide and adapt the parameters of a wireless transmission based on the load of the operating environment can be applied to hospital settings where various e-health applications have different levels of priority, and can be modeled as protected users or as primary/secondary users. Thus, to avoid any Electromagnetic Interference (EMI), medical devices are designated as protected users while telemedicine applications are the primary users (PUs) with higher priorities than those of the hospital information systems (used for medical records) that are considered secondary users (SUs). By far, the most important function in a CR network is spectrum sensing: SUs have to detect the availability of the spectrum and the presence of PUs. Three other functions are also required: Spectrum management that estimates the availability of the spectrum for SUs, spectrum sharing that distributes the spectrum gaps evenly among all SUs, and spectrum mobility that ensures same quality of transmission while moving from one spectrum gap to another. Since spectrum sensing is the most important function, especially in a licensed band scenario (e.g., hospital setting), we emphasize few crucial aspects of sensing that must be addressed. The first thing to be established is what needs to be sensed by SUs. In [65] there is a good summary of the spectrum parameters that can be detected (i.e., frequency, time, location, spreading code if used by PU, and angle). Second, equally important, is the algorithm used for sensing and the sensing method (e.g., cooperative sensing - centralized, distributed or external [65]). One possible classification of sensing techniques is: Transmitter detection (matched filter detection, energy detection, or feature detection), cooperative detection, and interference-based detection [60]. A graphic comparison of their accuracies and complexities is given in [65].

One other way to mitigate the issues related to EMI in a hospital setting is through a short range communication technology (UWB) as the communication channel between the sensor nodes and the PD. This requirement stems from the fact that both ZigBee and Bluetooth standards used as radio interfaces for WBAN sensor nodes have scalability problems and interfere with WLANs widely used by many medical devices in the operating room (OR). In addition to that, there is a demand for transmitting higher data rates for the more advanced medical tools (e.g., real-time capsule endoscopy). These sensors can have transmission rates from less than 100 bits/s up to tens of Mbits/s. Both ZigBee and Bluetooth are limited to data rates below $1 \mathrm{Mbit} / \mathrm{s}$. The most important aspect of UWB is that it does not cause significant inter- 
ference to other wireless standards, and is no threat to patients' health. Second, the nature of the wideband signal and the very low EIRP (Effective Isotropically Radiated Power) spectral density make the signal hard to jam. Finally, the high data rates reachable with this technology have no comparison with the ones achieved by narrow-band methods. Circuits and architectures for possible WBAN UWB implementations are described in [9] and [51], with an emphasis on the integration with CRs in [11].

\section{CONCLUSIONS}

This paper presented a large array of challenges and solutions related to the design and network communication aspects of WBANs. The purpose was not to explain in too much detail each topic, but rather to flag barriers and point out recent solutions as well as some future promising directions. In particular, we mentioned a possible evolution of WBANs within the realm of cognitive networks, where the crowded radio spectrum is more readily and fairly shared through flexible licensing, new techniques and technologies.

\section{REFERENCES}

[1] Ameen, M. A., Nessa, A., And Kwak, K. S. QoS issues with focus on wireless body area networks. In Proceedings of the International Conference on Convergence and Hybrid Information Technology (2008), pp. 801-807.

[2] Ashok, R. L., And Agrawal, D. P. Next-generation wearable networks. Computer 36, 11 (November 2003), 31-39.

[3] Baldus, H., Corroy, S., Klabunde, K., and Schenk, T. Human-centric connectivity enabled by body-coupled communications. IEEE Communications Magazine (June 2009), 172-178.

[4] Barakah, D. M., And Ammad-uddin, M. A survey of challenges and applications of wireless body area network (WBAN) and role of a virtual doctor server in existing architecture. In Proceeding of the 3rd IEEE ISMS 2012 (2012), pp. 214-219.

[5] Basagni, S., Naderi, M. Y., Petrioli, C., and Spenza, D. Wireless sensor networks with energy harvesting. In Mobile Ad Hoc Networking: Cutting Edge Directions, S. Basagni, M. Conti, S. Giordano, and I. Stojmenovic, Eds. John Wiley \& Sons, Inc., Hoboken, NJ, March 52013 , ch. 20, pp. 703-736.

[6] Braem, B., Latre, B., Moerman, I., Blondia, C., And Demeester, $\mathrm{P}$. The wireless autonomous spanning tree protocol for multihop wireless body area networks. In Proceedings of MobiQuitous 2006 (San Jose, CA, July 17-21 2006).

[7] Brito, M., Vale, L., Carvalho, P., And Henriques, J. A sensor middleware for integration of heterogeneous medical devices. In Proceedings of IEEE EMBS (Buenos Aires, Argentina, August 31-September 4 2010), pp. 5189-5192.

[8] Chaczko, Z., Kale, A., And Chiu, C. Intelligent health care - a motion analysis system for health practitioners. In Proceedings of ISSNIP 2010 (2010), pp. 303-308.

[9] Chandrakasan, A. P. ET AL. Low-power impulse radio architectures and circuits. Proceedings of the IEEE 97, 2 (Feb 2009), 332-352.

[10] Chavez-Santiago, R., and Balasingham, I. Cognitive radio for medical wireless body area networks. In Proceedings of IEEE CAMAD 2011 (2011), pp. 148-152.

[11] Chavez-Santiago, R., Khaleghi, A., Balasingham, I., And Ramstad, T. A. Architecture of an ultra wideband wireless body area network for medical applications. In Proceedings of IEEE ISABEL 2009 (November 22-27 2009), pp. 1-6.

[12] Chen, M., Gonzalez, S., Vasilakos, A., CaO, H., And Leung, V. C. M. Body area networks: A survey. ACM/Springer MONET 16 (2011), 171-193.
[13] Chiti, F., Fantacci, R., Archetti, F., Messina, E., And ToscAni, D. An integrated communications framework for context aware continuous monitoring with body sensor networks. IEEE Journal on Selected Areas in Communications 27, 4 (May 2009), 379-386.

[14] de Silva, B., Natarajan, A., And Motani, M. Inter-user interference in body sensor networks: Preliminary investigation and an infrastructure-based solution. In Proceedings of IEEE BSN 2009 (2009), pp. 35-40.

[15] Dionigi, M., Mongiardo, M., Sorrentino, R., and Tomassoni, C. Networks methods for wireless resonant energy links WREL computations. In Proceedings of ICEAA 2009 (2009).

[16] Edwards, R. M., AND KhatTaK, M. I. Understanding body-centric antennas. In Proceedings of the Loughborough Antennas \& Propagation Conference (Loughborough, UK, November 8-9 2010), pp. 629-631.

[17] FAnG, G. ET AL. Medical body area Networks: Opportunities, challenges and practices. In Proceedings of the IEEE ISCIT 2011 (2011), pp. 562-567.

[18] Galluccio, L., Melodia, T., Palazzo, S., and Santagati, G. E. Challenges and implications of using ultrasonic communications in intra-body area networks. In Proceedings of IEEE WONS 2012 (2012), pp. 182-189.

[19] Gopalan, S. A., And Park, J. T. Energy-efficient MAC protocols for wireless body area networks: Survey. In Proceedings of the International Congress on Ultra Modern Telecommunications and Control Systems and Workshops (2010), pp. 739-744.

[20] Gowrishankar, S., Basavaraju, T. G., Manjaiah, D. H., AND SARKAR, S. K. Issues in wireless sensor networks. In Proceedings of the World Congress on Engineering (London, UK, July 2-4 2008).

[21] Gu, L., And Stankovic, J. A. Radio-triggered wake-up capability for sensor networks. In Proceedings of the 10th Real-time and Embedded Technology and Applications Symposium (2004).

[22] Gupta, A., and Abhayapala, T. D. Body area networks: Radio channel modeling and propagation characteristics. In Proceedings of AusCTW 2008 (2008), pp. 58-63.

[23] Gupta, B., Valente, D., Cianca, E., and Prasad, R. FM-UWB for radar and communications in medical applications. Applied Sciences on Biomedical and CommunicationTechnologies (2008), 1-5.

[24] Hanson, M. A., Powell, H. C., Barth, A. T., Ringgenberg, K., Calhoun, B. H., Aylor, J. H., And LACH, J. Body Area Sensor Networks: Challenges and Opportunities. IEEE Computer Society (2009), 58-65.

[25] Hewitt, C. A. ET AL. Multilayered carbon nanotubes/polymer composite based termoelectric fabrics. American Chemical Society-Nano Letters (January 2012).

[26] Hollick, M., Martinovic, I., Krop, T., and Rimac, I. A survey of dependable routing in sensor networks, ad hoc networks, and cellular networks. In Proceedings of IEEE EUROMICRO 2004 (2004).

[27] Hovakeemian, Y., NAik, K., And Nayak, A. A survey on dependability in body area networks. In Proceedings of ISMICT 2011 (2011), pp. 10-14.

[28] Hoyt, R. W., Reifman, J., Coster, T. S., and Buller, M. J. Combat medical informatics: Present and future. In Proceedings of AMIA 2002 (2002), pp. 335-339.

[29] Hu, L., And Evans, D. Localization for mobile sensor networks. In Proceedings of ACM MobiCom 2004 (September 26-October 1 2004), pp. 1-13.

[30] Hussain, M. A., Alam, N., Ullah, S., Ullah, N., and KWAK, K. TDMA based directional MAC for WBAN. In Proceeding of IEEE INC 2010 (2010).

[31] Istepanaian, R. S. H., Sungoor, A., Faisal, A., And Philip, N. Internet of M-health Things 'm-IoT'. In Proceedings of the IET Assisted Living Conference (London, April 6 2011). 
[32] Istepanaian, R. S. H., And Zhang, Y. T. Guest editorial introduction to the special section: $4 \mathrm{G}$ Health-The Long-term Evolution of M-Health. IEEE Transactions on Information Technology in Biomedicine 16, 1 (January 2012), 1-5.

[33] Jovanov, E. Wireless technology and system integration in body area networks for M-health applications. In Proceedings of 27th IEEE Annual Conference on Engineering in Medicine and Biology (Shanghai, China, September 1-4 2005), pp. 7158-7160.

[34] Jovanov, E., O'Donnell Lords, A., Raskovic, D., Cox, P. G., Adhami, R., And Andrasik, F. Stress monitoring using a distributed wireless intelligent sensor system. IEEE Engineering in Medicine and Biology (May-June 2003), $49-55$

[35] Juels, A., And Wattenberg, M. A fuzzy commitment scheme. In Proceeding of CCS '99 (Singapore, November 1-4 1999), pp. 28-36.

[36] KaO, J. C., AND Marculescu, R. On optimization of e-textile systems using redundancy and energy-aware routing. IEEE Transactions on Computers 55 (2006), $745-756$.

[37] Kolar, V. Challenges in directional antennas. Master's thesis, Binghamton University - State University of New York, Binghamton, NY, 2004.

[38] KuRs, A. ET AL. Wireless power transfer via strongly coupled magnetic resonances. Science 317 (July 2007).

[39] Kwon, H. T., And LeE, S. Energy-efficient multi-hop transmission in body area networks. In Proceedings of IEEE PIMRC 2009 (2009), pp. 2142-2146.

[40] Latre, B., Braem, B., I., M., Blondia, C., Reusens, E., Joseph, W., And Demeester, P. A low-delay protocol for multihop wireless body area networks. In Proceedings of MobiQuitous 2007 (2007).

[41] Lukowicz, P., Amft, O., Roggen, D., And Cheng, J. On-body sensing: From gesture-based input to activity-driven interaction. IEEE Computer 43 (October 2010), 92-96.

[42] Marculescu, D. ET AL. Electronic textiles: A platform for pervasive computing. Proceedings of IEEE 91, 12 (December 2003), 1995-2018.

[43] Mitcheson, P. D. Energy harvesting for human wearable and implantable bio-sensors. In Proceedings of IEEE EMBS 2010 (Buenos Aires, Argentina, August 31-September 4 2010), pp. 3432-3436.

[44] Motani, M., Yap, K. K., Natarajan, A., and de Silva, B. Network characteristics of urban environments for wireless BAN. In Proceedings of IEEE BIOCAS 2007 (2007), pp. 179-182.

[45] Oliveira, C., Mackowiak, M., and Correia, L. M. Challenges for body area networks concerning radio aspects. In Proceedings of European Wireless 2011 (April 2011), pp. $77-81$.

[46] Olugbara, O. O., Adigun, M. O., Ojo, S. O., And MudALI, P. Utility grid computing and body area network as enabler for ubiquitous rural e-healthcare service provisioning. In Proceedings of the IEEE 9th International conference on e-Health Networking, Application and Services (2007), pp. 202-207.

[47] Patwari, N., Ash, J. N., Kyperountas, S., Hero, A., Moses, R. L., and Correal, N. S. Locating the nodes - cooperative localization in wireless sensor networks. IEEE Signal Processing Magazine (July 2005), 54-69.

[48] Phunchongharn, P., Hossain, E., Niyato, D., And Camorlinga, S. A cognitive radio system for E-health applications in a hospital environment. IEEE Wireless Communications (February 2010), 20-28.

[49] Poon, C. Y., Zhang, Y. T., and Bao, S. D. A Novel Biometrics Method to Secure Wireless Body Area Sensor Networks for Telemedicine and M-Health. IEEE Communications Magazine (April 2006), 73-81.
[50] Ramli, S. N., And Ahmad, R. Surveying the wireless body area network in the realm of wireless communication. In Proceeding of IEEE IAS 2011 (2011), pp. 58-61.

[51] RyCKAERT, J. E. A. Ultra-wide-band transmitter for low-power wireless body area networks: Design and evaluation. IEEE Transactions on Circuits and Systems 52, 12 (December 2005), 2515-2525.

[52] Santagati, G. E., Melodia, T., Galluccio, L., and PAlazzo, S. Distributed MAC and rate adaptation for ultrasonically networked implantable sensors. In Proceedings of IEEE SECON 2013 (New Orleans, LA, June 24-27 2013).

[53] Schenk, T. C. W., Mazloum, N. S., Tan, L., And Rutten, P. Experimental characterization of the body-coupled communications channel. In Proceedings of IEEE ISWCS 2008 (2008), pp. 234-239.

[54] Taherkordi, A., Taleghan, M. A., And Sharifi, M. Dependability considerations in wireless sensor networks applications. Journal of Networks 1, 6 (2006), 28-35.

[55] Takahashi, D., XiaO, Y., and Hu, F. LTRT: Least total-route temperature routing for embedded biomedical sensor networks. In Proceedings of IEEE GLOBECOM 2007 (2007), pp. 641-645.

[56] Taylor, C., And Pasquale, J. Towards a proximal resource-based architecture to support augmented reality applications. In Proceedings of the Cloud-Mobile Convergence for Virtual Reality Workshop (Waltham, MA, March 20 2010), pp. 5-9.

[57] Teng, X. F., Zhang, Y. T., Poon, C. C. Y., And Bonato, P. Wearable medical systems for p-health. IEEE Reviews in Biomedical Engineering 1 (2008), 62-74.

[58] Tseng, Y. C. ET AL. A wireless human motion capturing system for home rehabilitation. In Proceedings of $M D M$ 2009 (May 18-20 2009), pp. 359-360.

[59] Venkatasubramanian, K. K., Banerjee, A., and Gupta, S. K. S. Plethysmogram-based secure inter-sensor communication in body area networks. In Proceedings of IEEE Milcom 2008 (2008), pp. 1-7.

[60] WANG, W. Spectrum sensing for cognitive radio. In Proceedings of the 3rd International Symposium on Intelligent Information Technology Application Workshops (2009), pp. 410-412.

[61] Wang, Y., LiU, X., And Yin, J. Requirements for Quality of Service in Wireless Sensor Networks. In IEEE Computer Society - Proceedings of ICNICONSMCL (2006).

[62] Wegmueller, M. S., Kuhn, A., Froehlich, J., Oberle, M., Felber, N., And Fichtner, W. An attempt to model the human body as a communication channel. IEEE Transactions on Biomedical Engineering 54, 10 (2007), 1851-1857.

[63] Winterhalter, C. A. ET AL. Development of electronic textiles to support networks, communications, and medical applications in future u.s. military protective clothing systems. IEEE Transactions on Information Technology in Biomedicine 9, 3 (September 2005), 402-406.

[64] Wu, X., D'Auriol, B. J., Cho, J., and Lee, S. Optimal routing in sensor networks for in-home health monitoring with multi-factor considerations. In Proceedings of IEEE PerCom 2008 (2008), pp. 720-725.

[65] YuCEK, T., AND ARsLAN, H. A survey of spectrum sensing algorithms for cognitive radio applications. IEEE Communications Surveys \& Tutorials 11, 1 (2009), 116-130.

[66] Zasowski, T., Althaus, F., Stager, M., Wittneben, A., And Troster, G. UWB for noninvasive wireless body area networks: Channel measurements and results. Proceedings of the Conference on Ultra Wideband Systems and Technologies (2003), 285-289.

[67] Zasowski, T., Meyer, G., Althaus, F., And Wittneben, A. Propagation effects in UWB body areal networks. In Proceedings of the IEEE conference on Ultra-wideband (2005), pp. 16-21. 